\title{
Minor shift in background substitutional patterns in the Drosophila saltans and willistoni lineages is insufficient to explain GC content of coding sequences
}

\author{
Nadia D Singh*1, Peter F Arndt ${ }^{2}$ and Dmitri A Petrov ${ }^{1}$
}

\author{
Address: ${ }^{1}$ Department of Biological Sciences, Stanford University, 371 Serra Mall, Stanford, CA 94305, USA and ${ }^{2}$ Max Planck for Molecular \\ Genetics, 14195 Berlin, Germany \\ Email: Nadia D Singh* - ndsingh@stanford.edu; Peter F Arndt - arndt@molgen.mpg.de; Dmitri A Petrov - dpetrov@stanford.edu \\ * Corresponding author
}

Published: 18 October 2006

BMC Biology 2006, 4:37 doi:10.1186/1741-7007-4-37
Received: 08 May 2006

Accepted: 18 October 2006

This article is available from: http://www.biomedcentral.com/I741-7007/4/37

(c) 2006 Singh et al; licensee BioMed Central Ltd.

This is an Open Access article distributed under the terms of the Creative Commons Attribution License (http://creativecommons.org/licenses/by/2.0), which permits unrestricted use, distribution, and reproduction in any medium, provided the original work is properly cited.

\begin{abstract}
Background: Several lines of evidence suggest that codon usage in the Drosophila saltans and $D$. willistoni lineages has shifted towards a less frequent use of GC-ending codons. Introns in these lineages show a parallel shift toward a lower GC content. These patterns have been alternatively ascribed to either a shift in mutational patterns or changes in the definition of preferred and unpreferred codons in these lineages.
\end{abstract}

Results and discussion: To gain additional insight into this question, we quantified background substitutional patterns in the saltans/willistoni group using inactive copies of a novel, $Q$-like retrotransposable element. We demonstrate that the pattern of background substitutions in the saltans/willistoni lineage has shifted to a significant degree, primarily due to changes in mutational biases. These differences predict a lower equilibrium GC content in the genomes of the saltans/ willistoni species compared with that in the $D$. melanogaster species group. The magnitude of the difference can readily account for changes in intronic GC content, but it appears insufficient to explain changes in codon usage within the saltans/willistoni lineage.

Conclusion: We suggest that the observed changes in codon usage in the saltans/willistoni clade reflects either lineage-specific changes in the definitions of preferred and unpreferred codons, or a weaker selective pressure on codon bias in this lineage.

\section{Background}

Codon bias refers to the unequal usage of synonymous codons in protein coding sequences. Preferred codons are believed to correspond to more abundant transfer (t)RNAs [1]. In genes with high codon bias, the frequent presence of preferred codons is believed to increase the efficiency and/or fidelity of translation [2-4]. In Drosophila, empirical evidence suggests that the degree of codon bias can have substantial effects on active protein level; replacements of preferred codons with unpreferred ones in the alcohol dehydrogenase (Adh) gene result in significant decreases in levels of protein activity [5].

Within the genome of a particular species, the level of codon bias for any given gene is thought to be governed by the dynamic interplay among natural selection, mutation, and drift. Accordingly, codon bias levels will be affected by the rate and dominance of mutations affecting 
codon bias, the stochasticity associated with the population processes affecting these mutations (mediated through factors such as effective population size and recombination rate), and the strength of natural selection on codon bias. Variation in codon usage within a single genome is often associated with variation in one or more of these factors. In Drosophila melanogaster, for instance, codon bias is positively correlated with gene expression, which is thought to reflect increased selective benefits of translational efficiency for highly expressed genes [6-8]. Likewise, the negative correlation between codon bias and protein length in this species may result from Hill-Robertson effects [9], or may occur because increases in codon bias have stronger effects in short genes than in long genes [7,10-12]. The increase in codon bias associated with increased recombination rate in D. melanogaster is likely due to a recombination-associated substitutional bias [13-15], but Hill-Robertson effects may also play a role [16-18].

The genome hypothesis [19] suggests that the definition of "preferred" and "unpreferred" codons is the same for all genes in the genome, as codon preferences likely reflect tRNA abundances. Changes in codon usage patterns between species may be due to the same forces that modulate codon bias within any particular genome, but may also result from shifts in the definitions of preferred and unpreferred codons. Such shifts are not very common in evolution, and are generally observed only among very distantly related organisms. Within Drosophila, for instance, D. melanogaster and D. pseudoobscura, which diverged 25-40 million years ago (MYA) [20], have nearly identical definitions of major and minor codons [21].

In contrast, species in the D. saltans/willistoni lineages, which diverged from D. melanogaster around 30-40 MYA [20], show a substantially different pattern of codon usage from that found in the melanogaster and obscura group species. The saltans/willistoni lineages diverged from $D$. melanogaster prior to the split of the melanogaster and obscura groups but after the split of the Drosophila and Sophophora subgenera (Figure 1). Phylogenetic analysis [22] suggests that species within the melanogaster and obscura groups show roughly the ancestral pattern of codon usage, while there has been a significant reduction in the usage of G- or C-ending codons in the common ancestor of the saltans and willistoni lineages. In these species, the reduction of GC content is evident not only at third codon positions but also, albeit to a lesser extent, in introns and at first codon positions [22-26].

One hypothesis for the pronounced change in GC content in coding sequences is that the definitions of preferred and unpreferred codons have changed in the saltans/willistoni group [27]. This hypothesis is supported by the obser- vation that some amino acids show a greater change in GC content than do others [27]. However, a change in codon preferences cannot be invoked to explain the change in base composition of noncoding sequences.

One explanation for the concomitant shifts in GC content of codon and noncoding sequences is a genome-wide shift in the pattern of background substitutions [15] in favor of lower GC content in the saltans/willistoni group $[22,25,28]$. Such a shift could arise from either a change in mutational patterns or an increased rate of fixation of As and Ts mediated by biased gene conversion or natural selection. However, it is difficult to reconcile a simple substitutional model with the observed heterogeneity of change in GC content among amino acids.

To help distinguish between these two competing hypotheses, we investigated background patterns of nucleotide substitution in nine species belonging to the saltans/willistoni clade. We inferred these patterns by investigating rates of nucleotide substitution in nonfunctional fragments of a novel $Q$-like retrotransposable element. Our results suggest a minor shift in the spectrum of background nucleotide substitution in saltans/willistoni group relative to that found in D. melanogaster. The magnitude of this shift to is sufficient to generate a reduction of $11.5 \%$ at most in the GC content in the saltans/willistoni group. This is consistent with the reduction in GC content of intronic sequences in this lineage, but contrasts with the observed reduction in GC content of coding sequences of $\sim 30 \%$. Consequently, we suggest that the change in noncoding GC content in the saltans/willistoni group may result from background substitutional patterns, whereas the change in GC content of coding sequences reflects either novel codon preferences or a weakening of selection on codon bias.

\section{Results \\ Identification of Q-saltans I}

We isolated a novel retrotransposable element in the $D$. saltans genome using degenerate PCR. This element showed highest homology with a $Q$ element originally found in the Anopheles gambiae genome [29], and accordingly, we named this novel retrotranspon Q-saltans1. Using PCR primers specific to Q-saltans1, we identified a total of 58 paralogous copies of Q-saltans1 from individual flies from nine species in the saltans and willistoni species groups. Evolutionary relationships among the nine species studied are presented in Figure 1[28]. Ten copies of $Q$-saltans 1 were isolated from $D$. saltans, six from $D$. prosaltans, seven from $D$. subsaltans, seven from $D$. neocordata, eight from $D$. emarginata, five from $D$. tropicalis, five from $D$. equinoxialis, five from $D$. paulistorum and five from $D$. willistoni. 


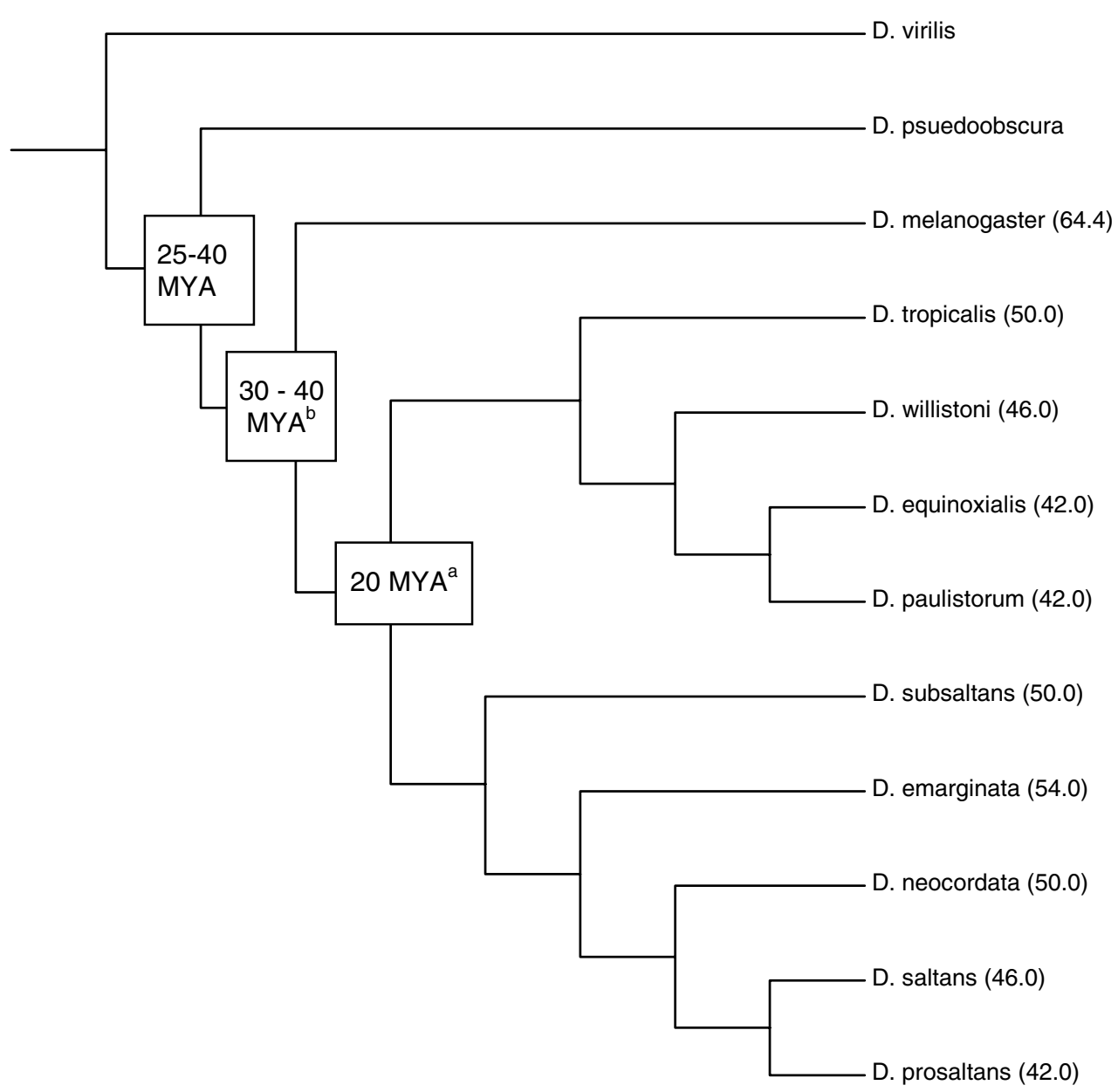

Figure I

Evolutionary relationships based on Xdh among the nine species studied including D. melanogaster, D. pseudoobscura and D. virilis as outgroups [26]. GC content at third position sites are presented in parentheses [23, 28]. D. melanogaster diverged from $D$. pseudoobscura 25-40 MYA [20], D. melanogaster and the saltans/willistoni groups diverged approximately 30-40 MYA [20], while the saltans and willistoni groups diverged approximately 20 MYA [27]

\section{Phylogenetic reconstruction in D. saltans}

We built a phylogenetic tree using parsimony criteria to reconstruct the evolutionary relationships among the 58 paralogs of Q-saltans1 (Figure 2). We mapped all 515 unambiguous changes to individual branches of the phylogeny. Changes mapping to the internal branches of the phylogeny are shared among elements, which suggests that they correspond to the constrained evolution of the active lineages of Q-saltans1 [30]. Substitutions on terminal branches are unique to individual paralogs and, provided sampling is sufficiently dense, should reflect the neutral evolution of the dead-on-arrival fragments commonly generated by non-long terminal repeat (LTR) elements [30,31]. In this model, substitutions on the internal branches should show substantially more third-position changes compared with the first and second positions, while substitutions on the terminal branches should fall into these classes with equal probability. For internal branches, we did indeed observe a sharp overabundance of third-position changes ( 81 third-position changes compared with 45 other (first + second) position changes, $P$ $<<0.001$, G-test). In contrast, we observed 199 other (first + second) changes and 107 third position changes on the terminal branches, which is not significantly different from the expected $2: 1$ ratio $(P=0.67, \mathrm{G}$-test). We used the identified terminal branch substitutions for all subsequent analyses.

\section{Intraspecific variability of the rates of nucleotide substitution in D. melanogaster}

To test for a shift in the pattern of background nucleotide substitution in the saltans/willistoni group relative to that of D. melanogaster, we compared background substitutional patterns derived from phylogenetic analyses of 
inactive copies of transposable elements (Q-saltans1 in the saltans/willistoni group versus the retrotransposable element Helena [32] and the non-autonomous DNA element DNAREP1_DM [15] in D. melanogaster). To ensure that comparisons between the patterns estimated from Qsaltans1 and the D. melanogaster transposable elements truly reflect interspecific differences, we first ascertained the amount of variability in the substitutional patterns that could result from differences in (i) the identity of the elements and their specific sequences, (ii) the genomic location of these transposable elements, and (iii) potential errors of phylogenetic reconstruction.

To assess variation in background substitutional patterns associated with different types of nonfunctional elements and their genomic location, we compared the substitutional profiles inferred from three types of transposable elements in D. melanogaster (Figure 3A). Two of the pro-

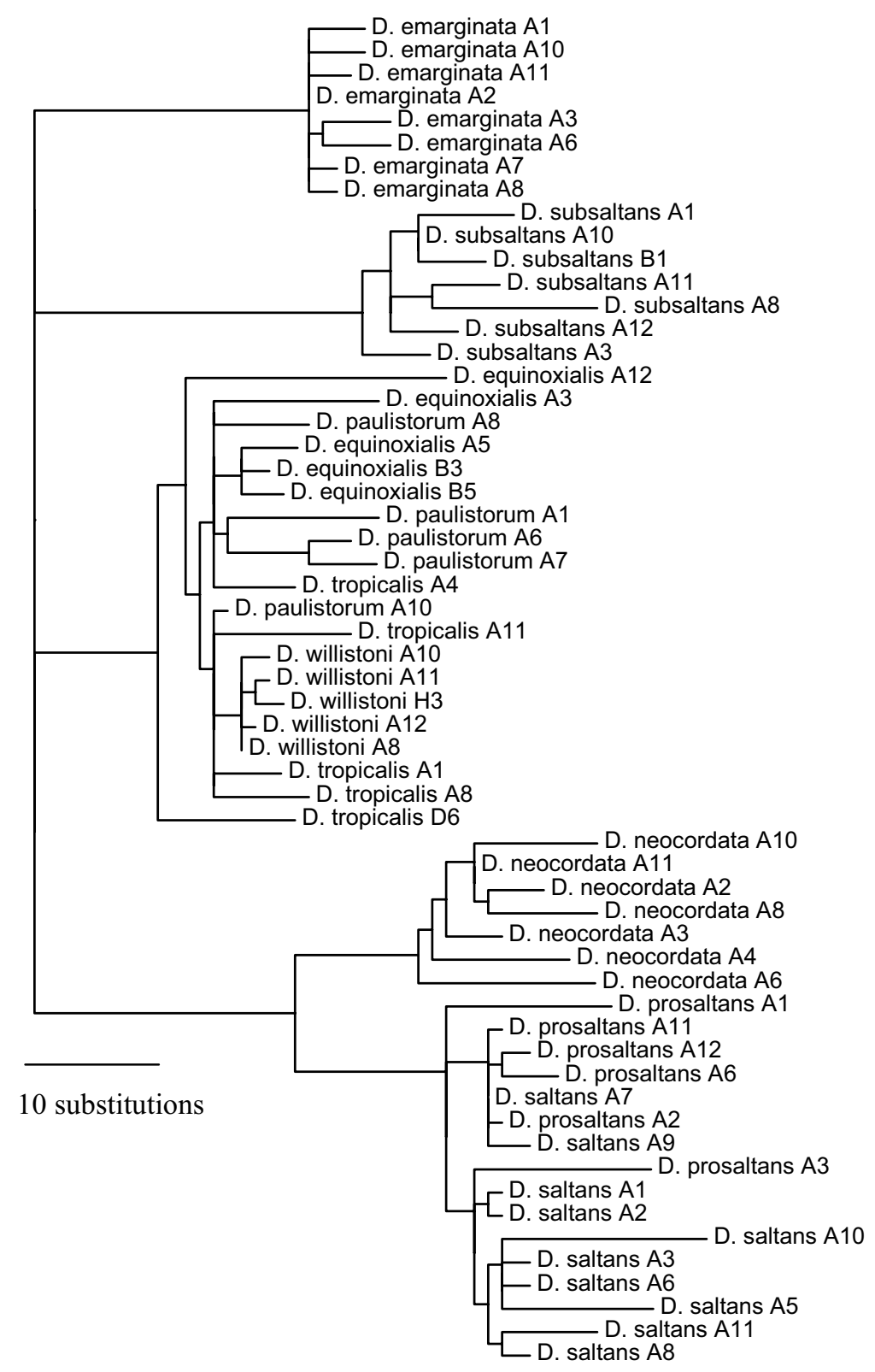

Figure 2

Phylogeny of the fifty-eight paralogous copies of the $Q$-like retrotransposon isolated from the $D$. saltans and $D$. willistoni lineages. Branch lengths are proportional to the number of substitutions incurred along each branch. 
A)

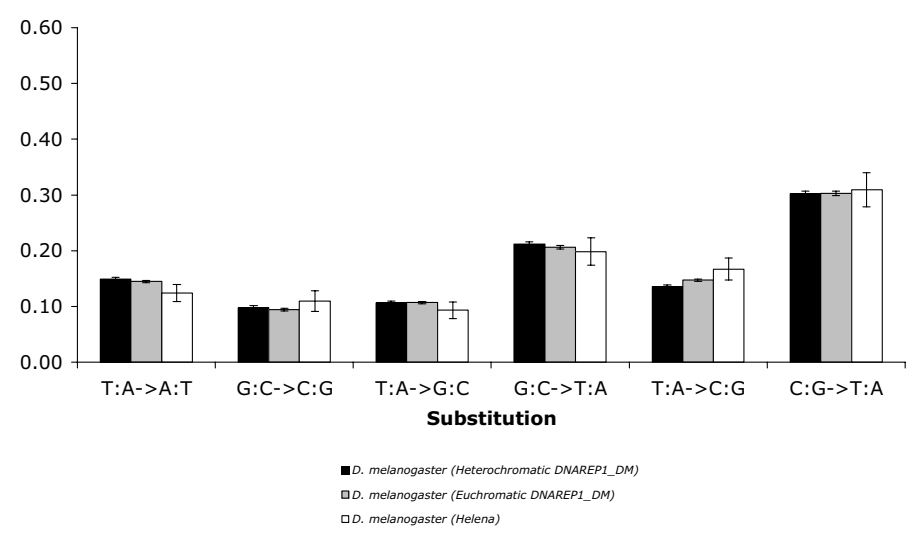

B)

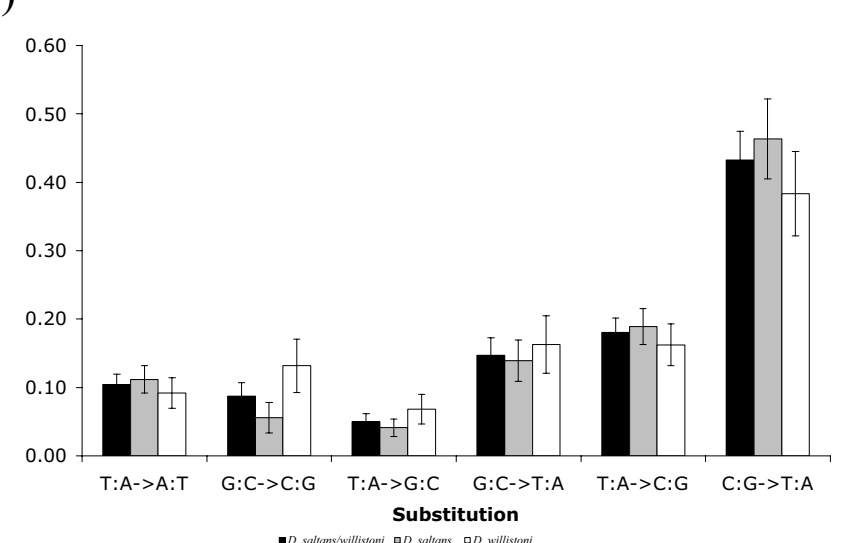

C)

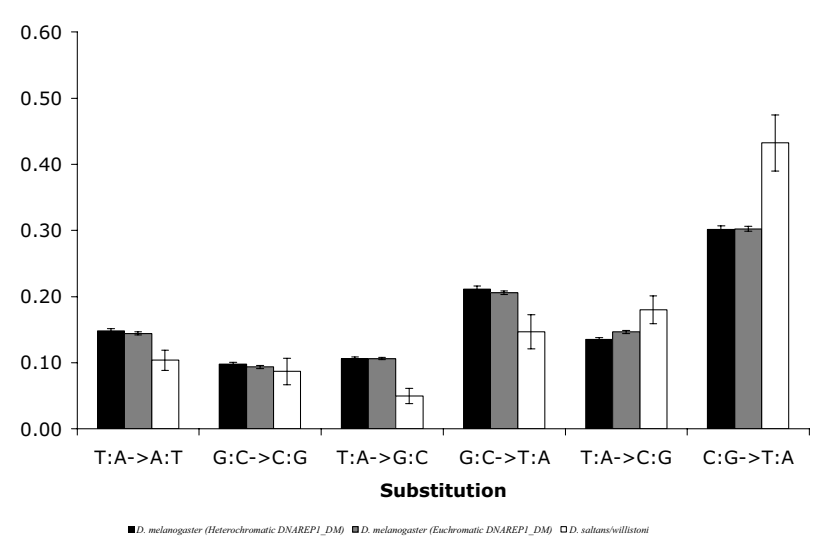

Figure 3

Relative rates of each of the six pairs of complementary nucleotide substitution in $A$ ) different noncoding elements from $D$. melanogaster, B) the saltans/willistoni group combined and D. saltans and D. willistoni and separately and C) in D. melanogaster and in the combined saltans/willistoni group. Three datasets were used to estimate relative rates of nucleotide substitution in D. melanogaster (see Materials and Methods). The rate of each pair of complementary nucleotide substitutions is depicted, normalized by the total substitution rate. Error bars represent the standard deviation due to sampling error only. 
A)

B)

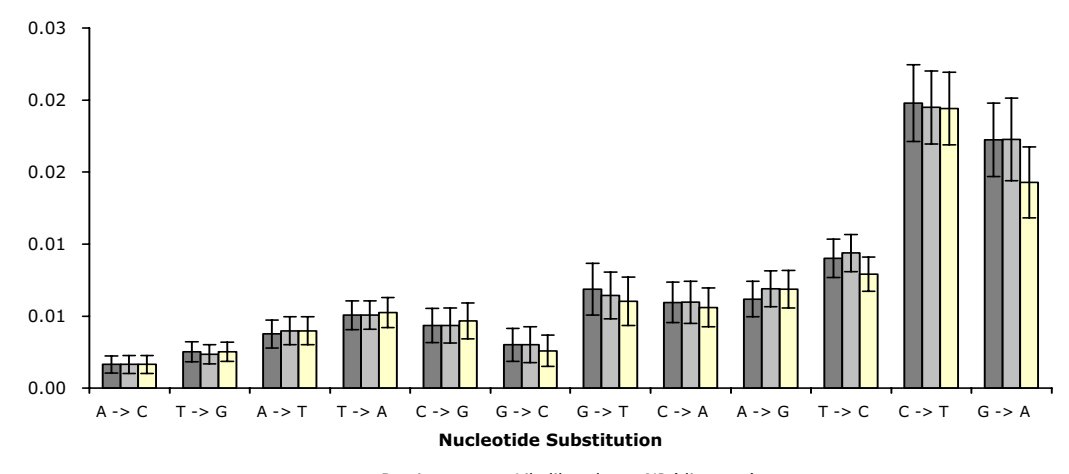

口Parsimony םLikelihood םNJ (distance)

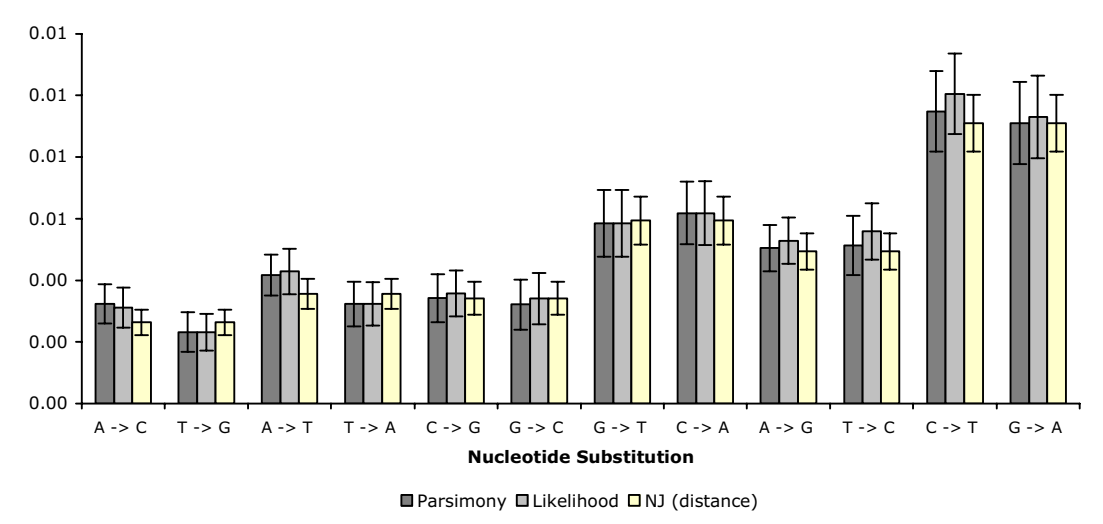

Figure 4

Relative rates of each of the twelve nucleotide substitutions inferred from alternative methods of phylogenetic reconstruction for A) Q-like elements in D. saltans and willistoni and B) Helena elements from D. melanogaster.

files were inferred from patterns of nucleotide substitution in DNAREP1_DM [15]; we present the spectrum of single-nucleotide substitutions assuming a star phylogeny for both heterochromatic and euchromatic elements [15]. The third estimate of background substitutional patterns in D. melanogaster was inferred from the terminal branch substitutions of the maximum parsimony tree for Helena [32]. In each of these three cases, we normalized the estimate of each of the six rates by the estimate of the total substitution rate (which is a function of the mutation rate and of the age of the individual elements), resulting in the estimate of the relative rates of single-nucleotide substitution.

The only significant difference among these three types of data was in the rate of the T:A $\rightarrow$ C:G transition, which was significantly lower in heterochromatic sequences of DNAREP1_DM compared with euchromatic fragments of this element $(P=0.002$, two-tailed $t$-test $)$. However, the magnitude of the difference in the rate of this transition is quite small (Figure 3A, Table 2). Equilibrium GC content inferred from the patterns of substitutions does vary between heterochromatic and euchromatic sequences of D. melanogaster, although the magnitude of the difference is quite small, on the order of 3\% [15]. Overall, however, the substitutional profiles estimated using heterochromatic fragments of DNAREP1_DM, euchromatic fragments of DNAREP1_DM, and nonfunctional fragments of the retrotransposon Helena are very similar.

To estimate the sensitivity of the patterns of substitution to errors in phylogenetic reconstruction, we compared rates of nucleotide substitution based on terminal branch substitutions of maximum parsimony, maximum likelihood (HKY85) and neighbor-joining (distance) trees. We explored this potential sensitivity to phylogenetic reconstruction in both the Q-saltans1 elements and the Helena elements. For both transposable elements, there were no significant differences in rates of individual nucleotide substitutions $(P>0.2$, all pairwise comparisons, two- 
tailed $t$-test) (Figure 4A and 4B). In addition, comparisons of equilibrium GC revealed no significant differences $(P>$ 0.4 , all pairwise comparisons, two-tailed $t$-test).

These results give us confidence that variability in the identity and genomic location of the studied transposable elements or potential errors of phylogenetic reconstructions do not significantly affect estimated patterns of single-nucleotide substitution for these data. Therefore, the comparison of patterns of substitution derived from $Q$ saltans1 and from the D. melanogaster transposable elements should primarily reflect interspecific differences in the patterns of nucleotide substitution. In all subsequent analyses, we used the maximum parsimony tree for $Q$ saltans 1 and Helena and a star phylogeny assumed for DNAREP1_DM elements.

\section{Comparisons of substitutional profiles between saltans and willistoni lineages}

The shift in the pattern of nucleotide substitutions is hypothesized to have occurred prior to the split of the saltans and willistoni groups. We thus tested whether the patterns of substitution are indeed similar in the saltans and willistoni species groups. To do so, we identified terminal branch substitutions on the maximum parsimony tree of Q-saltans1, and separated those substitutions occurring on branches leading to paralogs isolated from a species in the saltans group from those occurring on branches leading to a paralog isolated from a species in the willistoni group. These two classes of terminal branch substitutions were then used to infer patterns of substitution specific to the saltans and willistoni groups, respectively.

The estimated relative rates of single-nucleotide substitutions are presented in Figure 3B. None of the six substitutions was significantly different in pairwise comparisons $(P>0.09$, all comparisons, two-tailed $t$-test $)$. This homogeneity in background substitutional patterns justifies aggregation of all of the terminal branch substitutions on the Q-saltans1 tree to estimate the overall pattern of singlenucleotide substitutions in the saltans/willistoni lineage.

\section{Comparisons of substitutional profiles between $\mathbf{D}$. melanogaster and saltans/willistoni lineages}

For simplicity, we present the comparison of the saltans/ willistoni substitutional profile with the most robust $D$. melanogaster estimates, those derived from heterochro- matic and euchromatic fragments of DNAREP1_DM. The resulting profiles are presented in Figure $3 \mathrm{C}$ and the comparisons of rates are presented in Table 1. Two nucleotide substitution rates are significantly different between the D. melanogaster (both in euchromatin and heterochromatin) and saltans/willistoni lineages. The relative rate of T:A $\rightarrow \mathrm{G}: \mathrm{C}$ transversion is significantly lower, whereas the rate of $\mathrm{C}: \mathrm{G} \rightarrow \mathrm{T}: \mathrm{A}$ transition is significantly higher in the saltans/willistoni groups (Bonferroni-corrected $P<0.0001$ and $P<0.03$, respectively, both comparisons, two-tailed $t$ test). In addition, we have tentative support for lower rates of T:A $\rightarrow$ A:T and G:C $\rightarrow$ T:A substitutions in the saltans/ willistoni lineages, although these differences are not significant after correcting for multiple tests $(P<0.025$, Bonferroni-corrected $P>0.05$, both comparisons, two-tailed $t$ test). We also estimated predicted equilibrium GC content $\left(\mathrm{GC}^{*}\right)$ in both lineages based on the rates of nucleotide substitutions, which revealed that GC* in the saltans and willistoni groups $(28.4 \%)$ is lower than that in heterochromatic and euchromatic fragments of D. melanogaster (32.0\% and $33.3 \%$, respectively), although not significantly so (Bonferroni-corrected $P>0.1$, both comparisons, one-tailed $t$-test). GC* estimated from transitions only, while lower in D. saltans/willistoni (29.4\%), was not significantly different from comparable estimates in $D$. melanogaster (30.9\% and $32.7 \%$ in heterochromatic and euchromatic sequences, respectively) (Bonferroni-corrected $P>0.2$, both comparisons, one-tailed $t$-test).

This analysis was carried out using the combined data from DNAREP1_DM fragments taken from low-recombination and high-recombination portions of the euchromatic fraction of the D. melanogaster genome. The aggregation of these data is justified given the lack of statistically significant differences in equilibrium base composition between these regions [15]. However, the best estimate of equilibrium GC content in high-recombination regions of $D$. melanogaster euchromatin is higher than that in low-recombination regions, and thus we might be underestimating the magnitude of the shift in GC content in the saltans/willistoni lineages. Importantly, equilibrium GC content (GC*) estimated from fragments of DNAREP1_DM in regions of high recombination (35.0\%) is also not significantly different from GC* in the saltans/ willistoni lineages (Bonferroni-corrected $P>0.05,1$-tailed $t$-test).

Table I: Relative rates of single-nucleotide substitutions (with standard errors) in D. melanogaster and $D$. saltans/willistoni

\begin{tabular}{|c|c|c|c|c|c|c|}
\hline & $\mathrm{T}: \mathrm{A}->\mathrm{A}: \mathrm{T}$ & $\mathrm{G}: \mathrm{C}->\mathrm{C}: \mathrm{G}$ & $\mathrm{T}: \mathrm{A}->\mathrm{G}: \mathrm{C}$ & $\mathrm{G}: \mathrm{C}->\mathrm{T}: \mathrm{A}$ & $\mathrm{T}: \mathrm{A}->\mathrm{C}: \mathrm{G}$ & C:G->T:A \\
\hline D. melanogaster (Heterochromatic DNAREPI_DM) & $0.15(0.004)$ & $0.10(0.003)$ & $0.11(0.003)$ & $0.21(0.005)$ & $0.13(0.003)$ & $0.30(0.005)$ \\
\hline D. melanogaster (Euchromatic DNAREPI_DM) & $0.14(0.002)$ & $0.09(0.002)$ & $0.11(0.002)$ & $0.21(0.003)$ & $0.15(0.002)$ & $0.30(0.004)$ \\
\hline D. saltans/willistoni (Q-saltans I) & $0.10(0.016)$ & $0.09(0.020)$ & $0.05(0.012)$ & $0.15(0.026)$ & $0.18(0.021)$ & $0.43(0.043)$ \\
\hline
\end{tabular}


Table 2: Ratio of rates of significantly different single-nucleotide substitutions between $D$. saltans/willistoni and $D$. melanogaster

\begin{tabular}{|c|c|c|c|c|c|c|}
\hline & $\mathrm{T}: \mathrm{A}->\mathrm{C}: \mathrm{G} \mathrm{r}$ & C:G->T:A & $\begin{array}{l}\text { Minimum fixation } \\
\text { bias* }\end{array}$ & $\mathrm{T}: \mathrm{A}->\mathrm{G}: \mathrm{C}$ & $\mathrm{G}: \mathrm{C}->\mathrm{T}: \mathrm{A}$ & Minimum fixation bias* \\
\hline $\begin{array}{l}\text { Ratio of rates in saltans/willistoni and D. } \\
\text { melanogaster (Euchromatic DNAREPI_DM) }\end{array}$ & $1.23(0.13)^{* *}$ & $1.43(0.12)$ & $\begin{array}{c}N_{e} s(\text { mel })=-4.76 \\
N_{e} s(\text { saltans })=- \\
4.85\end{array}$ & $0.47(0.10)$ & $0.71(0.11)$ & $\begin{array}{c}N_{\mathrm{e}} s(\mathrm{mel})=2.86 \\
N_{\mathrm{e}} s(\text { saltans })=2.98\end{array}$ \\
\hline $\begin{array}{l}\text { Ratio of rates in saltans/willistoni and } D \text {. } \\
\text { melanogaster (Heterochromatic } \\
\text { DNAREPI DM) }\end{array}$ & $1.33(0.13)$ & $1.43(0.12)$ & undefined & $0.21(0.003)$ & $0.15(0.002)$ & undefined \\
\hline
\end{tabular}

* Fixation bias is measured in the effective strength of natural selection (product of effective population size and selection coefficient) that would generate such a bias. Positive sign implies preference for A:T pairs and negative sign implies preference toward G:C pairs.

** The number in parentheses is the standard error.

\section{Are differences in mutation rates or differences in the probabilities of fixation responsible for the differences in substitution rates between these lineages?}

The observed changes in nucleotide substitutions may result from changes either in the mutation rates or in the probability of fixation of different mutations. To distinguish between these two possibilities, we employed the forward-reverse test [33]. In the case of the comparisons of the saltans/willistoni rates with the rates derived from euchromatic DNAREP1_DM elements in D. melanogaster, the $95 \%$ confidence limits of the ratios of the forward and reverse substitutions do allow for the theoretical possibility of the differences in fixation biases explaining the patterns (Table 2). However, the forward-reverse analysis indicates that such differences in fixation probabilities require $\mathrm{G}: \mathrm{C}$ nucleotides to be very strongly preferred when we consider changes in the rates of transitions ( $\mid$ Nes $\mid>4$ in both lineages) and strongly unpreferred when we consider changes in the rates of transversions ( $|\mathrm{Nes}|>2$ in both lineages) (Table 2). On balance, a shift in mutational patterns appears to be a more likely explanation.

\section{Discussion \\ Estimating patterns of substitution using dead-on-arrival copies of non-LTR elements}

Previous data on the base composition of coding and coding sequences suggest that GC content in the saltans/willistoni group differs from GC content in D. melanogaster [22$25,27,28]$. The shift in GC content in coding sequences has been argued to result from novel codon preferences in this species group [27]. However, the observation that base composition of noncoding sequences shows a shift in GC content in the same direction (although to a lesser degree) has been used as evidence in favor of a mutational shift $[22,25,28]$. To distinguish among these possibilities, we assayed background patterns of nucleotide substitution in unconstrained sequences. This method of using inactive fragments of transposable elements has been used previously to investigate patterns of background point substitution and insertion/deletions in Drosophila and other organisms $[15,30,34]$.
To estimate background patterns of single-nucleotide substitutions, we identified a novel non-LTR element in $D$. saltans. We cloned and sequenced 58 paralogous copies of this Q-like retrotransposon (Q-saltans1) in nine species from the willistoni and saltans groups. We analyzed substitutions that map to the terminal branches of the phylogenetic trees reconstructed using these sequences, and extracted estimates of the individual rates of single-nucleotide substitutions using a maximum-likelihood procedure $[35,36]$.

The pattern of single-nucleotide substitutions estimated from this procedure conforms to our expectations of unconstrained sequence evolution. Changes were equally likely in all three codon positions, giving us confidence that we indeed observe background patterns of substitution using these sequences. Moreover, our estimates of the spectrum of single-nucleotide substitution are robust to errors in phylogenetic reconstruction. Comparing the profile of background substitutional patterns derived from various methods of phylogenetic reconstruction (maximum parsimony, maximum likelihood, and neighborjoining) yielded no significant differences; in all cases the estimates of individual rates of single-nucleotide substitutions were virtually identical $(P>0.2$, all comparisons, two-tailed $t$-test).

\section{Comparison of the patterns of substitution in dead-on- arrival copies of $\mathbf{Q}$-saltans I and inactive copies of $\mathbf{D}$. melanogaster transposable elements}

Patterns of background substitution revealed by the analysis of the inactive copies of Q-saltans1 appeared similar for saltans and willistoni groups (Figure $3 \mathrm{~B}$ ), which is consistent with the shift in GC content occurring prior to the separation of these lineages $[22,25]$. Therefore, we combined all of the Q-saltans1 data to estimate the overall pattern of background substitution in the saltans/willistoni lineage.

The pattern of nucleotide substitution in D. melanogaster was estimated using two different transposable elements: Helena [30] and DNAREP1_DM [15]. The profiles of sin- 
gle-nucleotide substitutions estimated from these different elements are strikingly similar (Figure 3A), suggesting that the species-specific pattern of background substitution is largely insensitive to the identity of the transposable element studied. While small in magnitude, there do appear to be heterogeneities in background substitutional patterns associated with genomic location [15], and accordingly, we treated euchromatic and heterochromatic copies of DNAREP1_DM separately in our analysis.

Overall, the saltans/willistoni pattern is quite similar to the patterns estimated in D. melanogaster (Figure 3C) and D. virilis [30]. In all cases, the most frequent substitution is the $\mathrm{C}: \mathrm{G} \rightarrow \mathrm{T}: \mathrm{A}$ transition, whereas the reverse transition ( $\mathrm{T}: \mathrm{A} \rightarrow \mathrm{C}: \mathrm{G}$ ) occurs at rates similar to that of transversions [32]. Moreover, the G:C $\rightarrow$ T:A transversion appears to be most frequent transversions in both $D$. melanogaster and the saltans/willistoni group.

However, the saltans/willistoni pattern does differ in two respects from the pattern observed in D. melanogaster. There is a statistically significant increase in the rate of C:G $\rightarrow \mathrm{T}: \mathrm{A}$ transitions and a significant decrease in the rate of the $\mathrm{T}: \mathrm{A} \rightarrow \mathrm{G}: \mathrm{C}$ transversion in the willistoni/saltans lineage. Notably, both of these changes lead to a reduction in the overall GC content in saltans/willistoni lineages. However, the expected equilibrium GC content based on the saltans/ willistoni pattern $(28.4 \%)$ shifts only modestly (roughly 4-7\%) compared with that estimated using DNAREP1_DM $(32.0 \%, 33.3 \%$ and $35.0 \%$ for heterochromatic, low-recombination euchromatic, and highrecombination euchromatic sequences, respectively). Given the errors on our estimates of $\mathrm{GC}^{*}$ for both species, we can say with $95 \%$ confidence that the magnitude of the difference in $\mathrm{GC}^{*}$ between these two groups is at most $11.5 \%$ and could in fact be nonexistent.

\section{Distinguishing between mutational generation and fixation probability models}

A background substitutional bias can be mediated through a bias either in the generation of novel mutations, or in the fixation probabilities of novel mutations.
Note, however, that if the shift toward higher rates of G:C $\rightarrow \mathrm{T}: \mathrm{A}$ and $\mathrm{C}: \mathrm{G} \rightarrow \mathrm{T}: \mathrm{A}$ substitutions in the saltans/willistoni lineages was due entirely to the decrease of the probability of fixation of G:C alleles at the expense of A:T alleles (either due to the effects of natural selection or biased gene conversion), we would then also expect to see a concomitant decrease in the rate of the reverse (T:A $\rightarrow \mathrm{G}: \mathrm{C}$ and $\mathrm{T}: \mathrm{A} \rightarrow \mathrm{C}: \mathrm{G}$ ) substitutions in the saltans/willistoni lineage. However, this does not appear to be the case for the rates of $\mathrm{C}: \mathrm{G} \rightarrow \mathrm{T}: \mathrm{A}$ and $\mathrm{T}: \mathrm{A} \rightarrow \mathrm{C}: \mathrm{G}$ transitions (both of which are increased in the saltans/willistoni lineage), or for $\mathrm{T}: \mathrm{A} \rightarrow \mathrm{G}: \mathrm{C}$ and G:C $\rightarrow$ T:A transversions (both of which are both decreased in the saltans/willistoni lineage) (Figure 3C).

We employed a more rigorous test to distinguish between mutational variation and fixation biases for the shift in background substitutional patterns in D. saltans/willistoni. The forward-reverse test [33] suggests that while fixation biases can in principle explain the difference in substitutional patterns between D. melanogaster and D. saltans/ willistoni, this model would require novel G:C mutations to be strongly preferred when generated by a transition, while being strongly unpreferred when generated by a transversion. This eliminates the possibility of differences in the strength of natural selection acting on GC content being responsible for these patterns (as it cannot distinguish between A:T versus G:C polymorphisms generated by transitions or transversions). This leaves only the possibility of differences in the strength of biased gene conversion. However, the strength of the putative bias in rates of gene conversion seems extreme and appears implausible, requiring for instance that the rates of forward and reverse mutations be different by over a hundred-fold [33]. Thus, the most probable explanation for the observed differences in substitution patterns in D. saltans/ willistoni is a change in the rates of mutation.

Table 3: Percent GC of coding and noncoding sequences from $D$. willistoni, $D$. saltans and $D$. melanogaster

\begin{tabular}{|c|c|c|c|c|c|c|c|c|c|}
\hline & $A d h^{\mathrm{a}}$ & Soda & Per & $X d h^{\mathrm{a}}$ & Amyre/a $^{a}$ & $D d c^{a}$ & $G p d h^{\mathrm{a}}$ & $\begin{array}{l}\text { Short } \\
\text { Introns }\end{array}$ & $\begin{array}{l}\text { Long } \\
\text { Introns }\end{array}$ \\
\hline D. saltans & 52.6 & 45.9 & $54.9 \mathrm{~b}$ & 43.5 & & & & $27.1^{c}$ & $30.6 \mathrm{c}$ \\
\hline D. willistoni & 54.1 & 52.1 & $60.5^{\mathrm{a}}$ & 47.2 & 48.5 & 52.9 & 51.6 & $32.9 c$ & $35.5^{c}$ \\
\hline $\begin{array}{l}\text { D. } \\
\text { melanogaster }\end{array}$ & 81.5 & 76.0 & $80.8^{a}$ & 64.4 & 68.2 & 73.6 & 76.5 & $34.5^{d}$ & $37.1^{d}$ \\
\hline
\end{tabular}

aPercent GC at third position sites as reported by Rodriguez-Trelles et al. [22]

bPercent GC at third position sites as reported by Rodriguez-Trelles et al. [23]

cPercent GC from combined analysis of RpL32, Adh, Hsp83, Sod, Gpdh, Amyrel, Ddc, per and ry, as reported by Powell et al. [27]

dPercent GC from 225 introns in D. melanogaster, as reported by Hadrill et al. [38] 


\section{Role of novel background substitutional patterns in modulating base composition of coding and noncoding sequences}

Although we have documented a minor shift in background substitutional patterns in the saltans and willistoni species groups, the extent to which this shift is reflected in the base composition of coding and noncoding DNA remains to be seen. Note that, given the divergence time between $D$. melanogaster and D. saltans/D. willistoni of approximately 30-40 million years [20] and the rates of neutral point substitution in Drosophila [37], we would expect that base composition of unconstrained sequences in D. saltans and D. willistoni genomes should have approached the equilibrium values corresponding to the derived patterns of point substitution in the saltans/willistoni clade.

The documented shift in base composition of intronic sequences is similar between short and long introns, and appears to be minor. For D. melanogaster, D. saltans and D. willistoni, the GC content of short introns is slightly lower than that of long introns (Table 3). Base composition shifts in D. saltans and willistoni to similar degrees in both intron classes towards $\sim 7 \%$ lower GC content in D. saltans and $\sim 2 \%$ lower GC content in D. willistoni $[27,38]$. The magnitude of this shift in the base composition of intronic sequences is entirely consistent with our estimates of the change in patterns of background substitution, which could generate a difference in GC content of up to $\sim 12 \%$, although it is likely to be smaller.

In contrast, GC content in coding sequences, particularly at third-position sites, in the saltans/willistoni group show a much greater depression in GC content relative to $D$. melanogaster than do introns [22-26] (Table 1). On average, GC content at third-position sites in D. saltans and $D$. willistoni is $23.6 \%(13-30 \%)$ lower than that at the same sites in D. melanogaster. The magnitude of this change in GC content is incompatible with our estimates of background substitutional patterns, which suggests that other evolutionary forces must be at play.

However, we do believe that our documented shift in substitutional biases may serve to modulate base composition at coding sites to some degree. Indeed, it is intriguing that the amino acids with the most significant shifts in codon usage are those two-fold amino acids encoded by $\mathrm{C} / \mathrm{U}$-ending codons, as the relative rate of $\mathrm{C}: \mathrm{G} \rightarrow \mathrm{T}: \mathrm{A}$ substitutions has increased significantly in this lineage (Figure 3C). However, given that $\mathrm{GC}^{*}$ estimated using all substitutions is very similar to $\mathrm{GC}^{*}$ estimated using only transitions, it is not likely that this single pair of complementary nucleotide substitutions is wholly responsible for the exaggerated response of the two-fold degenerate amino acids.
The dramatic shift in base composition in coding sequences might very well reflect novel codon preferences in this species group, as suggested by others [27]. In support of this model is the observation that some amino acids show more marked changes in codon usage than other amino acids in this clade [27], which is seemingly at odds with a mutational model given that almost all preferred codons in D. melanogaster are G- or C-ending. In addition, assuming that orthologous genes in D. melanogaster and D. saltans/willistoni are under similar selection pressures for codon bias, the changing codon preference model predicts that genes with the most highly biased patterns of codon usage will experience the greatest deviation in codon usage patterns relative to D. melanogaster, which is precisely what is observed [27].

It is important to note that there are two other forces that may be contributing to the evolution of base composition of coding and noncoding sequences in the saltans and willistoni lineages. First, transcription-associated mutational biases appear to be operating in several systems (for review see Aguilera [39]). Were such a bias operating in Drosophila, this could also shape GC content of genic sequences in this system; however, the concordance between the expected GC content based on background substitutional patterns and the observed GC content of intronic sequences suggests that transcription-associated mutational biases are unlikely to play a significant role in the evolution of base composition of genic sequences in the saltans/willistoni lineages. Moreover, comparisons of background substitutional patterns in transcribed and untranscribed sequences in D. melanogaster also show no evidence of transcription-associated mutational biases [15].

Second, the marked reduction in GC content of exonic sequences in D. saltans and willistoni could also result from a relaxation of natural selection on codon bias in these lineages. This could in principle result from a reduction in effective population size, but this model would require a severe and prolonged bottleneck in the lineage leading to the saltans/willistoni species group, or concomitant reductions in effective population size for all species in this group. Alternatively, weaker selection on codon bias in the saltans/willistoni group could reflect lineagespecific fitness effects for synonymous mutations. As is the case in D. saltans and D. willistoni, several other species in the D. melanogaster subgroup show significant changes in codon bias in the absence of marked base-composition heterogeneity of nearby noncoding sequences, which has been argued to result from either a relaxation of selection on codon bias mediated through lineage-specific changes in effective population size, from fitness effects, $[10,40]$ or from positive selection [41]. 
Like the changing codon preference model, a general model of relaxation of selection on codon bias in $D$. saltans/willistoni would also predict that the genes with the highest codon bias would experience the greatest deviation in codon usage in $D$. saltans/willistoni relative to $D$. melanogaster. Formally distinguishing between the shifting codon preference and relaxation of selection models by specifically comparing codon usage preferences in lowly and highly expressed genes between $D$. melanogaster and $D$, willistoni at a larger scale will soon become possible, once the D. willistoni genome has been assembled and annotated.

\section{Conclusion}

Analysis of substitutional patterns inferred from nonfunctional fragments of transposable elements in the Drosophila saltans/willistoni lineage provides evidence in support of a minor shift in patterns of nucleotide substitution towards decreased GC content of at most $11.5 \%$. These novel patterns of nucleotide substitution are likely modulating the base composition of unconstrained sequences in the genome, and are sufficient to account for documented shift in GC content of noncoding sequences such as introns. The base composition of sequences under selective constraint such as coding sequences, however, while governed in part by substitutional biases, appear to be primarily modulated by other evolutionary forces. In this particular instance, one likely explanation for the reduction in GC content in coding sequences in the saltans/willistoni group is a shift in codon preferences combined with an altered substitutional profile. However, general relaxation of selective pressure on codon bias in this species group is also possible, and further investigation will likely reveal the relative strengths of substitutional biases and natural selection in the determination of base composition of functional sequences.

\section{Methods}

\section{Drosophila strains}

Strains of Drosophila saltans, D. tropicalis, D. equinoxialis, D. paulistorum and $D$. willistoni were kindly provided by E. Baldal. D. saltans and D. willistoni samples were collected on the Donato Trail on Barro Colorado Island (BCI), Panama, D. tropicalis was collected in old forest in BCI, D. equinoxialis was collected on the Foster trail in Gigante, Panama, and D. paulistorum was collected from the Summit Botanical Gardens in Panama. We also purchased strains 14041-0831.0, 14042-0841.3, 14044-0872.0, and 14045-0901.0 from the University of Arizona Center for Insect Science Stock center, which correspond to D. neocordata, D. emarginata, D. subsaltans, and D. prosaltans, respectively.

\section{Genomic DNA extraction}

We extracted genomic DNA from single individuals taken from these nine strains of Drosophila according to protocol described by Greg Gloor and William Engels (personal communication). Each fly was crushed with the end of a pipette tip and subsequently immersed in a buffered solution (10 mM Tris-HCl pH 8.2, 1 mM EDTA, $25 \mathrm{mM} \mathrm{NaCl}$, $200 \mu \mathrm{g} / \mathrm{mL}$ proteinase $\mathrm{K}$ ). This was incubated at $37^{\circ} \mathrm{C}$ for 30 minutes, and then at $95^{\circ} \mathrm{C}$ for 2 minutes to inactivate the proteinase $\mathrm{K}$.

\section{Species confirmation}

To confirm the species assignment for each of the nine strains, we amplified and sequenced a portion of the xanthine dehydrogenase $(X d h)$ gene (rosy in D. melanogaster). The region of $X d h$ studied is approximately 725 bp in size and includes part of exon II in addition to intron $\mathrm{B}$, the distribution of which is confined to the saltans and willistoni lineages [42]. Primer sequences were: XDHF: 5' -CGTTCYTTGGTWGTWAGYC-3' and XDHR: $5^{\prime}$ GCAAAGGCYTCCTCCACATT-3'.

Amplifying conditions for the $X d h$ regions for each of the nine species are as follows: $94^{\circ} \mathrm{C}$ for $2 \mathrm{~min}, 35$ cycles of $94^{\circ} \mathrm{C}$ for $30 \mathrm{~s}, 52^{\circ} \mathrm{C}$ for $30 \mathrm{~s}, 72^{\circ} \mathrm{C}$ for 30 s, and a final extension of $72^{\circ} \mathrm{C}$ for $7 \mathrm{~min}$. All PCR reactions were $20 \mu \mathrm{l}$, and each contained $10 \mu \mathrm{l}$ ReadyMix REDTaq PCR Reaction Mix with $\mathrm{MgCl}_{2}$ (Sigma-Adrich, St Louis, MO, USA), $1 \mu \mathrm{l}$ of each $20 \mu \mathrm{M}$ primer, $7 \mu \mathrm{H}_{2} \mathrm{O}$, and $1 \mu$ genomic DNA. PCR reactions were enzymatically cleaned with exonuclease I and shrimp alkaline phosphatase, and were cycle-sequenced in half-strength half-reactions with Big Dye terminator mix (Applied Biosystems) under standard cycling conditions. These reactions were precipitated using ethanol and sodium acetate and sequenced on an ABI 377 sequencer (Applied Biosystems). Sequences were compared with the available sequences for this region in each species [28], with intron B serving as a diagnostic marker.

\section{Identification of non-LTR elements}

Degenerate primers designed for the reverse transcriptase domain of non-LTR retrotransposable elements in Arabidopsis were used to amplify potential retrotransposons in Drosophila saltans. Primer sequences were: DVO144: 5' GGGATCCNGGNCCNGAYGGNWT-3' and DVO145: 5' GGAATTCGGNSWNARNGGRYMNCCYTG-3' [43]. All PCR reactions were $20 \mu \mathrm{l}$, and each contained $10 \mu \mathrm{l}$ ReadyMix REDTaq PCR reaction mix with $\mathrm{MgCl}_{2}$ (SigmaAldrich), $0.8 \mu \mathrm{l}$ of $25 \mathrm{mM} \mathrm{MgCl}_{2}, 1.25 \mu \mathrm{l}$ of each $20 \mu \mathrm{M}$ primer, $5.7 \mu \mathrm{l} \mathrm{H}_{2} \mathrm{O}$, and $1 \mu \mathrm{l}$ genomic DNA. Cycling conditions were: $94^{\circ} \mathrm{C}$ for $2 \mathrm{~min}, 35$ cycles of $94^{\circ} \mathrm{C}$ for $1 \mathrm{~min}$, $47^{\circ} \mathrm{C}$ for $1 \mathrm{~min}, 72^{\circ} \mathrm{C}$ for $2 \mathrm{~min}$, and a final extension of $72^{\circ} \mathrm{C}$ for $15 \mathrm{~min}$. 
The pool of PCR products was cloned using a TOPO cloning kit (Invitrogen) for sequencing, and PCR (using vector primers T7 and M13R) was used to screen for colonies containing appropriately sized inserts. PCR products of interest were enzymatically cleaned with exonuclease I and shrimp alkaline phosphatase, and were cyclesequenced in half-strength half-reactions with Big Dye (Applied Biosystems) under standard cycling conditions. These reactions were precipitated using ethanol and sodium acetate and sequenced on an ABI 377 sequencer (Applied Biosystems).

These sequences were compared against sequences of all known non-LTR elements using BLASTX to identify clones containing inserts truly corresponding to retrotransposable elements. This technique yielded one clone containing a 271-bp insert with high homology to reverse transcriptases from other organisms, and has highest homology to the $Q$ retrotransposon isolated from Anopheles gambiae [29]; specific primers for this putative $Q$ element were designed from the sequence of this clone.

\section{Amplification of non-LTR elements in saltans and willistoni clades}

These element-specific primers were used to amplify paralogous copies of this $Q$ element in several species in the saltans and willistoni clades. Primer sequences are as follows: SaltansQF: 5' -CCGGATGGGATAGCTG-3' and SaltansQR: $\quad 5^{\prime}$-GGTTAGCGGTAGTAGATGTA-3'. Amplifications were carried out in $20 \mu \mathrm{l}$ reactions. For species in the saltans clade, each PCR reaction contained $10 \mu \mathrm{l}$ ReadyMix REDTaq PCR reaction mix with $\mathrm{MgCl}_{2}$ (Sigma), $1 \mu \mathrm{l}$ of each $20-\mu \mathrm{M}$ element-specific primer, $7 \mu \mathrm{l} \mathrm{H} \mathrm{H}_{2} \mathrm{O}$, and $1 \mu \mathrm{l}$ genomic DNA. Cycling conditions were: $94^{\circ} \mathrm{C}$ for 2 min, 35 cycles of $94^{\circ} \mathrm{C}$ for $1 \mathrm{~min}, 47^{\circ} \mathrm{C}$ for $1 \mathrm{~min}, 72^{\circ} \mathrm{C}$ for $2 \mathrm{~min}$, and a final extension of $72^{\circ} \mathrm{C}$ for $15 \mathrm{~min}$.

For species in the willistoni clade, two rounds of PCR were required for successful amplification. First, a $20-\mu \mathrm{l}$ reaction using DVO144 and DVO145 primers was carried out as described above. This PCR product was diluted 1:1000 and was used as template in a second round of PCR amplification using element-specific primers as described for the species in the saltans clade.

Products from PCR reactions using element-specific primers were cloned for each of the nine species under study. Colonies of interest were sequenced using T7 and M13R; paralogs differing by $<1 \%$ within an individual species were not included in the analysis.

\section{Analysis of background substitutional patterns}

In total, 58 distinct paralogous fragments $(271 \mathrm{bp}$ in length) of a non-LTR element were identified from the saltans and willistoni species groups. Sequences have been deposited to Genbank (under accession numbers AY920638-AY920695). We aligned the sequences using Sequencher software (version 3.1.1), and used PAUP software (Phylogenetic Analysis Using Parsimony; version $4.0 \mathrm{~b} 9)$ for the phylogenetic reconstruction of the relationships among the paralogous retrotransposons. Based on this phylogeny, we inferred the ancestral sequence at each terminal node; substitution frequencies were estimated by comparing those ancestral sequences with the sequences of the extant fragments.

The extant sequences show on average 5.2\% divergence from the ancestral sequences at their respective terminal nodes on the phylogeny. For the estimation of the substitution frequencies, we used a maximum-likelihood approach to include multiple and back substitutions within one branch at the same site. Importantly, we cannot capture multiple substitutions on different branches. However, owing to the low divergence among paralogs of Q-saltans1, the associated error due to such processes is likely to be quite small. All 12 possible single-nucleotide substitutions have been estimated. Details on a more general version of this approach including neighbor-dependent substitution processes have been discussed by Arndt $e t$ al. $[35,36]$. Once the 12 substitution frequencies are established, the stationary GC content $\left(\mathrm{GC}^{*}\right)$ can easily be computed [44].

Because we only have a finite amount of sequence data from which to estimate the substitution frequencies, these estimates will be affected by statistical errors; we can estimate these errors by bootstrapping our dataset. For a category with a total of $n$ aligned base pairs we resampled the data, drawing randomly and with replacement, $n$ pairs of aligned ancestral and daughter nucleotides. From this resampled sequence data, we estimated the substitution frequencies and the GC content as above. We repeated this resampling procedure $M$ times, and from the $M$ estimates of the above quantities calculated their standard deviation, which gave the statistical error due to the limited amount of sequence data. In our case, we found that $M=$ 500 samples is sufficient to estimate those errors [36].

\section{Background substitutional patterns in D. melanogaster}

Substitution patterns from D. melanogaster were inferred in three ways. Two profiles are based on autosomal copies of the element DNAREP1_DM [15], and we present data from elements found in both heterochromatic and euchromatic sequences. Although initial analysis of background substitutional patterns using DNAREP1_DM did distinguish between elements in high- versus low-recombination areas of the genome, there were no significant differences revealed between these two genomic locations [15]. As a result, these data were combined in the present analyses and are referred to as "euchromatic" elements. 
The pattern of substitutions does significantly differ between heterochromatic and euchromatic regions in $D$. melanogaster, but the differences are very small in magnitude [15].

We also present data from the retrotransposon Helena [45], which is based on 22 paralogs of this transposable element. These data were analyzed in the same way as the data from $D$. saltans and willistoni; phylogenies were reconstructed using PAUP, ancestral sequences at each terminal node were inferred, and substitution frequencies were estimated by comparing those ancestral sequences with the sequences of the extant fragments in a maximumlikelihood framework. Details on a more general version of the maximum-likelihood model used to estimate substitutional patterns in D. melanogaster including neighbordependent substitution processes have been discussed by Arndt et al. [35].

\section{Authors' contributions}

NDS, PFA, and DAP contributed to the development of this project idea, and the analysis and interpretation of the data presented in this report. NDS, PFA, and DAP were also responsible for the writing and editing of this manuscript, and have all given their final approval for its publication.

\section{Acknowledgements}

This work was supported in part by the Center for Evolutionary Studies at Stanford University (NDS) and the Stanford Genome Training Program funded by 5 T32 HG00044 from the NHGRI (NDS). We gratefully acknowledge E. Baldal for providing samples of several Drosophila species for analysis. Comments from two anonymous reviewers and an assistant editor improved the quality of this manuscript.

\section{References}

I. Shields DC, Sharp PM, Higgins DG, Wright F: 'Silent' sites in Drosophila genes are not neutral: evidence of selection among synonymous codons. Molec Biol Evol 1988, 5:704-716.

2. Akashi H, Kliman RM, Eyre-Walker A: Mutation pressure, natural selection, and the evolution of base composition in Drosophila. Genetica (Dordrecht) 1998, 102-103:49-60.

3. Akashi $\mathrm{H}$, Eyre-Walker A: Translational selection and molecular evolution. Current Opinion in Genetics \& Development 1998, 8:688-693.

4. Bulmer M: The selection-mutation-drift theory of synonymous codon usage. Genetics 1991, I 29:897-908.

5. Carlini DB, Stephan W: In Vivo Introduction of Unpreferred Synonymous Codons Into the Drosophila Adh Gene Results in Reduced Levels of ADH Protein. Genetics 2003, 163:239-243.

6. Bulmer M: Are codon usage patterns in unicellular organisms determined by selection mutation balance? Journal of Evolutionary Biology 1988, I: 15-26.

7. Duret L, Mouchiroud D: Expression pattern and, surprisingly, gene length shape codon usage in Caenorhabditis, Drosophila, and Arabidopsis. Proceedings of the National Academy of Sciences of the United States of America 1999, 96:4482-4487.

8. Sharp PM, Li WH: An evolutionary perspective on synonymous codon usage in unicellular organisms. Journal of Molecular Evolution 1986, 24:28-38.

9. Comeron JM, Kreitman M, Aguade M: Natural Selection on Synonymous Sites is Correlated with Gene Length and Recombination in Drosophila. Genetics 1999, 15 1:239-249.
10. Akashi H: Molecular Evolution Between Drosophila melanogaster and D. simulans: Reduced Codon Bias. Faster Rates of Amino Acid Substitution, and Larger Proteins in D. melanogaster. Genetics 1996, 144: 1297-1307.

II. Eyre-Walker A: Synonymous codon bias is related to gene length in coli Escherichia: Selection for translational accuracy? Molecular Biology and Evolution 1996, 13:864-872.

12. Marais G, Duret L: Synonymous codon usage, accuracy of translation, and gene length in Caenorhabditis elegans. Journal of Molecular Evolution 200 I, 52:275-280.

13. Marais G, Mouchiroud D, Duret L: Does recombination improve selection on codon usage? Lessons from nematode and fly complete genomes. Proceedings of the National Academy of Sciences of the United States of America 200I, 98:5688-5692.

14. Marais G, Mouchiroud D, Duret L: Neutral effect of recombination on base composition in Drosophila. Genetical Research 2003, $81: 79-87$

15. Singh ND, Arndt PF, Petrov DA: Genomic Heterogeneity of Background Substitutional Patterns in Drosophila melanogaster. Genetics 2004, 169:709-722.

16. Hey J, Kliman RM: Interactions between natural selection, recombination and gene density in the genes of Drosophila. Genetics 2002, 160:595-608.

17. Kliman RM, Hey J: Reduced natural selection associated with low recombination in Drosophila melanogaster. Molecular Biology and Evolution 1993, 10:1239-1258.

18. Marais G, Piganeau G: Hill-Robertson interference is a minor determinant of variations in codon bias across Drosophila melanogaster and Caenorhabditis elegans genomes. Molecular Biology and Evolution 2002, 19:1399-1406.

19. Grantham R, Gautier C, Gouy M, Mercier R, Pave A: Codon catalog usage and the genome hypothesis. Nucleic Acids Research 1980 , 8:r49-r62.

20. Powell JR, DeSalle R: Drosophila Molecular Phylogenies and Their Uses. Evolutionary Biology 1995, 28:87-I38.

21. Akashi H, Schaeffer SW: Natural selection and the frequency distributions of "silent" DNA polymorphism in Drosophila. Genetics 1997, 146:295-307.

22. Rodriguez-Trelles F, Tarrio R, Ayala FJ: Evidence for a high ancestral GC content in Drosophila. Molecular Biology and Evolution 2000, I7:1710-1717.

23. Rodriguez-Trelles F, Tarrio R, Ayala FJ: Switch in codon bias and increased rates of amino acid substitution in the Drosophila saltans species group. Genetics 1999, 153:339-350.

24. Anderson CL, Carew EA, Powell JR: Evolution of the Adh locus in the Drosophila willistoni group: The loss of an intron, and shift in codon usage. Molecular Biology and Evolution 1993, 10:605-618

25. Rodriguez-Trelles F, Tarrio R, Ayala FJ: Fluctuating mutation bias and the evolution of base composition in Drosophila. Journal of Molecular Evolution 2000, 50: I- 10.

26. Rodriguez-Trelles F, Tarrio R, Ayala FJ: Molecular evolution and phylogeny of the Drosophila saltans species group inferred from the Xdh gene. Molecular Phylogenetics and Evolution 1999, 13:|10-121

27. Powell JR, Sezzi E, Moriyama EN, Gleason JM, Caccone A: Analysis of a shift in codon usage in Drosophila. Journal of Molecular Evolution 2003, 57:S2 I4-S225.

28. Rodriguez-Trelles F, Tarrio R, Ayala FJ: Disparate evolution of paralogous introns in the $\mathbf{X d h}$ gene of Drosophila. Journal of Molecular Evolution 2000, 50:123-130.

29. Besansky NJ, Bedell JA, Mukabayire O: Q: A new retrotransposon from the mosquito Anopheles gambiae. Insect Molecular Biology 1994, 3:49-56.

30. Petrov DA, Lozovskaya ER, Hartl DL: High intrinsic rate of DNA loss in Drosophila. Nature (London) 1996, 384:346-349.

31. Luan DD, Korman MH, Jakubczak JL, Eickbush TH: Reverse transcription R2Bm RNA is primed by a nick at the chromosomal target site: a mechanism for non-LTR retrotransposition. Cell 1993, 72:595-605.

32. Petrov DA, Hartl DL: Patterns of nucleotide substitution in Drosophila and mammalian genomes. Proceedings of the National Academy of Sciences of the United States of America 1999, 96: 1475-1479.

33. Lipatov M, Arndt PF, Hwa T, Petrov DA: A Novel Method Distinguishes Between Mutation Rates and Fixation Biases in Pat- 
terns of Single-Nucleotide Substitution. Journal of Molecular Evolution 2006, 62:168-175.

34. Neafsey DE, Palumbi SR: Genome Size Evolution in Pufferfish: A Comparative Analysis of Diodontid and Tetraodontid Pufferfish Genomes. Genome Research 2003, 13:821-830.

35. Arndt PF, Petrov DA, Hwa T: Distinct changes of genomic biases in nucleotide substitution at the time of mammalian radiation. Molecular Biology and Evolution 2003, 20: 1887-1896.

36. Arndt PF, Hwa T: Identification and Measurement of Neighbor Dependent Nucleotide Substitution Processes. Bioinformatics 2005, $21: 2322-2328$.

37. Singh ND, Petrov DA: Rapid sequence turnover at an intergenic locus in Drosophila. Molec Biol Evol 2004, 21 :670-80.

38. Haddrill PR, Charlesworth B, Halligan DL, Andolfatto P: Patterns of intron sequence evolution in Drosophila are dependent on length and GC content. Genome Research 2005, 6:R67.

39. Aguilera A: The connection between transcription and genomic instability. EMBO (European Molecular Biology Organization) Journal 2002, 21:195-201.

40. Akashi H, Ko WY, Piao SF, John A, Goel P, Lin CF, Vitins AP: Molecular evolution in the Drosophila melanogaster species subgroup: Frequent parameter fluctuations on the timescale of molecular divergence. Genetics 2006, 172:17|I-1726.

41. DuMont VB, Fay JC, Calabrese PP, Aquadro CF: DNA variability and divergence at the Notch locus in Drosophila melanogaster and $D$. simulans: $A$ case of accelerated synonymous site divergence. Genetics 2004, 167:171-185.

42. Tarrio R, Rodriguez-Trelles F, Ayala FJ: New Drosophila introns originate by duplication. Proc Natl Acad Sci U S A 1998 95:1658-1662.

43. Wright DA, Ke N, Smalle J, Hauge BM, Goodman HM, Voytas DF: Multiple non-LTR retrotransposons in the genome of Arabidopsis thaliana. Genetics 1996, 142:569-578.

44. Arndt PF, Burge CB, Hwa T: DNA Sequence Evolution with Neighbor-Dependent Mutation. Journal of Computational Biology 2003, 10:313-322.

45. Petrov DA, Hartl DL: High rate of DNA loss in the Drosophila melanogaster and Drosophila virilis species groups. Molecular Biology and Evolution 1998, 15:293-302.

Publish with Bio Med Central and every scientist can read your work free of charge

"BioMed Central will be the most significant development for disseminating the results of biomedical research in our lifetime. "

Sir Paul Nurse, Cancer Research UK

Your research papers will be:

- available free of charge to the entire biomedical community

- peer reviewed and published immediately upon acceptance

- cited in PubMed and archived on PubMed Central

- yours - you keep the copyright
BioMedcentral 